\title{
The industrial evolution
}

\author{
Susannah J. Salter
}

This month's Genome Watch highlights new insights into the use of bacteria in industrial processes such as electricity generation and bioremediation.

Bacteria are used in many industrial processes, including food production, waste processing and the mass manufacture of biological products such as enzymes. Three recently published bacterial genomes offer insights into the potential utility of these bacteria in electricity generation and cleaning up chemical contaminants and oil spills.

Geobacter sulfurreducens, an anaerobic bacterium usually found in soil or aquatic sediment, is frequently exploited in microbial fuel cells, as it passes electrons out of the cell during anaerobic respiration. The electrons are transported along cytochrome-laden pili to reduce an external electron acceptor or cathode. A recent analysis comparing the genome of G. sulfurreducens str. KN400 (a novel strain) to that of G. sulfurreducens str. PCA (the type strain) explored the genetic basis of the improved electricity-generating phenotype of strain KN400 (REF. 1), which is due to faster turnover of redox reactions. Although this improvement was previously hypothesized to be caused by mutations in the genes relating to acetate oxidation or the synthesis of pili, the authors found that central energy metabolism genes were well conserved between the two

genomes. However, sequence differences

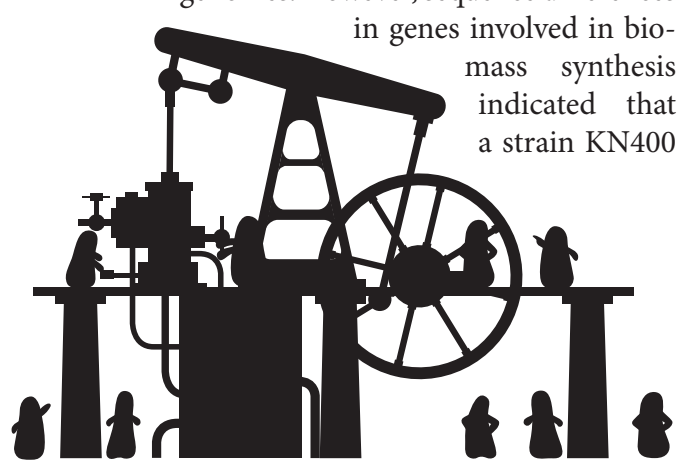

cell might need to oxidize more acetate than a strain PCA cell, with a consequent higher electron outflow, to achieve a comparable biomass yield.

The conductive pili of G. sulfurreducens str. KN400 are interesting in their own right, as this type of structure might one day be used as a template for nanoscale electronic 'wires'. Comparison of the two genomes revealed differences in the sequences of genes encoding two cytochrome proteins (which carry out electron transport) and a channel affecting the localization of these proteins along the length of the pilus. These differences might contribute to the improved electron transport observed in G. sulfurreducens str. KN400.

The genus Geobacter is also used in bioremediation - the use of microorganisms to break down environmental contaminants. Because Geobacter spp. reduce external metal electron acceptors, they can convert soluble metals such as iron III and uranium VI to insoluble forms, which then precipitate out of contaminated water. Geobacter lovleyi str. SZ not only precipitates uranium but also degrades the toxic chemical tetrachloroethene (PCE), a common groundwater contaminant used in the manufacture of hydrofluorocarbon refrigerants, for cleaning metals and in dry cleaning. A recent analysis of the 3.9 $\mathrm{Mb}$ chromosome of this strain provides evidence of substantial recent lateral gene transfer, possibly linked to the acquisition of a conjugative pilus ${ }^{2}$. As well as chromosomal changes, strain SZ has acquired plasmid pSZ77, containing a cobalamin biosynthesis cluster, which is important for the phenotype because cobalamin is required for the reductive dechlorination of PCE. The authors identified pSZ77-containing bacteria at geographically distant sites (South Korea and Canada) and demonstrated that cells carrying the plasmid cannot lose it. However, other species in the dechlorinating clade within the genus Geobacter do not carry the plasmid, so future work might reveal other adaptations for acquiring cobalamin.

Bioremediation can also be used in the clean-up of crude oil. In this area of research, ever more efficient mechanisms for degrading petroleum products are needed. The persistence of very-long-chain alkanes is a particular problem, as the sludge containing them can be difficult to recover. They are not easily biodegraded, although many microorganisms can break down short- to mediumchain alkanes using alkane hydroxylases. The draft genome of Pseudomonas aeruginosa str. SJTD-1 was recently published, and this strain was proposed as a new, environmentally adaptable candidate for cleaning up longchain alkanes ${ }^{3}$. The authors identified several putative alkane degradation enzymes in the $6.1 \mathrm{Mb}$ genome of $P$. aeruginosa str. SJTD-1, and analysis of the degradation mechanism is ongoing.

Bacterial skills have been put to work in all corners of industry, including power generation and environmental decontamination. Understanding the genetic variation that underlies these useful functions and the varying performances of different species is important for improving the efficiency of these systems and identifying new processes and applications in which they can be used.

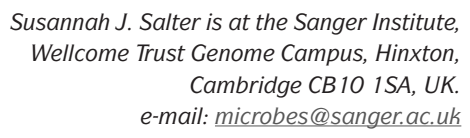
-mail:microbes@sanger.ac.uk

doi: 10.1038/nrmicro2915

1. Butler, J. E. et al. Comparative genomic analysis of Geobacter sulfurreducens KN400, a strain with enhanced capacity for electron transfer and electricity production. BMC Genomics 13, 471 (2012).

2. Wagner, D. D. et al. Genomic determinants of organohalide-respiration in Geobacter lovleyi, an unusual member of the Geobacteraceae. BMC Genomics 13, 200 (2012).

3. Liu, H. et al. Genome sequence of Pseudomonas aeruginosa strain SJTD-1, a bacterium capable of degrading long-chain alkanes and crude oil. J. Bacteriol. 194, 4783-4784 (2012)

Competing interests statement

The author declares no competing financial interests. 